\title{
Energy fluxes in helical magnetohydrodynamics and dynamo action
}

\author{
Mahendra K. Verma \\ Department of Physics, Indian Institute of Technology, Kanpur - 208016, INDIA
}

(Dated: October 19, 2002)

\begin{abstract}
Renormalized viscosity, renormalized resistivity, and various energy fluxes are calculated for helical magnetohydrodynamics using perturbative field theory. The calculation is to first-order in perturbation. Kinetic and magnetic helicities do not affect the renormalized parameters, but they induce an inverse cascade of magnetic energy. The sources for the the large-scale magnetic field have been shown to be (1) energy flux from large-scale velocity field to large-scale magnetic field arising due to nonhelical interactions, and (2) inverse energy flux of magnetic energy caused by helical interactions. Based on our flux results, a premitive model for galactic dynamo has been constructed. Our calculations yields dynamo time-scale for a typical galaxy to be of the order of $10^{8}$ years. Our field-theoretic calculations also reveal that the flux of magnetic helicity is backward, consistent with the earlier observations based on absolute equilibrium theory.
\end{abstract}

PACS numbers: PACS numbers: 47.27.Gs, 52.35.Ra, 91.25.Cw

\section{INTRODUCTION}

Generation of magnetic field in plasma, usually referred to as "dynamo", is one of the prominent and unsolved problems in physics and astrophysics. It is known that the magnetic field of galaxies, the Sun, and the Earth are neither due to some permanent magnet nor due to any remnants of the past, but it is generated by the nonlinear processes of plasma motion ([1, 2] and references therein). However, a solid quantitative understanding is lacking in this area inspite of various attempts for more than half century. There are various aspects in this problem, and we address energy transfer issues in this paper and in paper I ( $[3]$ ) using field-theoretic methods in a somewhat idealized environment, homogeneous and isotropic flows. In paper I we show that the nonhelical part of magnetohydrodynamic (MHD) interaction causes energy transfer from large-scale (LS) velocity field to large-scale (LS) magnetic field. In a typical dynamo environment however, the helical interactions cause an additional energy cascade of magnetic energy from small scales (SS) to large scales; the field-theoretic calculation of helical contribution to the energy flux is presented in this paper. Both helical and nonhelical factors contribute to the magnetic energy growth.

In the problem of magnetic field generation, it is required that the LS magnetic field is maintained at all time. There are several exact results in this area, e.g., dynamo does not exist in two dimensions as well as in axisymmetric flows [1]. In the past, several dynamos, e.g., rotor dynamo, 2-sphere dynamo etc., have been constructed [1], however, mean-field electrodynamics developed by Steenbeck et al. [4] (also see Krause and Rädler 2]) paved a way for practical calculations in astrophysical and terrestrial dynamo. This formalism also provided insights into the physical mechanism of dynamo, mainly that kinetic helicity $H_{K}=1 / 2(\langle\mathbf{u} \cdot \boldsymbol{\Omega}\rangle$, where $\mathbf{u}$ and $\boldsymbol{\Omega}$ are the velocity and vorticity fields respectively) plays an important role in the amplification of the magnetic field. The amplification parameter $\alpha_{u}$ was found to be $(\tau / 3) H_{K}$, where $\tau$ is the velocity de-correlation time.

Mean-field electrodynamics of Steenbeck et al. [4] is a kinematic theory. Here it is assumed that the velocity field is a known function, which is unaffected by the generated magnetic field. The later models which take into account the back reaction of the magnetic field to the velocity field are called dynamic models. One of the first dynamic model is due to Pouquet et al. 5] where they incorporated the feedback, and proposed that the modified $\alpha$ is proportional to residual helicity, $H_{K}-H_{J}$, where $H_{J}$ is the current helicity define as $1 / 2\langle\mathbf{b} \cdot \nabla \mathbf{b}\rangle$. Gruzinov and Diamond [ $[6]$ proposed a quenching mechanism where the amplification parameter was modified to

$$
\alpha=\frac{\alpha_{u}}{1+R_{m}(\bar{B} / \bar{U})^{2}}
$$

where $\bar{B}^{2}$ is the LS magnetic energy, $\bar{U}$ is the LS velocity field, and $R_{m}$ is the magnetic Reynolds number. Recently Field, Blackman, and Chou [7], and Chou [8] obtained a general expression for dynamic $\alpha$ coefficient as a function of Reynolds number and magnetic Prandtl number. Basu and Bhatthacharya [9] and Basu [10] have attempted to compute the dynamo coefficients $\alpha$ and $\beta$ using field-theoretic techniques.

In a recent development Brandenburg [20] investigated dynamo problem in isotropic and helical MHD. The the system is forced with kinetic helicity, they find magnetic energy transfer to large-scales. They indentify this mechanism, named as nonlinear alpha-effect, for magnetic energy growth. Magnetic helicity play an important role in this mechanism; we will discuss these issues in later part of the paper.

There are many numerical simulations of dynamo in various geometries. In simulations MHD equations are numerically solved with appropriate boundary conditions. In this paper we will only refer to the simulations performed for 
a periodic box; this is to avoid the complications of spherical geometry (e.g., effects of Coriolis force etc.). Pouquet et al. [5] numerically integrated the MHD equations on the basis of Eddy-damped-quasi-normal-Markovian (EDQNM) approximation. When kinetic energy $(\mathrm{KE}=1 / 2\langle\mathbf{u} \cdot \mathbf{u}\rangle)$ and $H_{K}$ were injected near a wavenumber band, both magnetic energy $(\mathrm{ME}=1 / 2\langle\mathbf{b} \cdot \mathbf{b}\rangle)$ and absolute value of magnetic helicity $\left|H_{M}\right|$ were found to increase. Magnetic helicity $H_{M}$ is defined as $1 / 2\langle\mathbf{a} \cdot \mathbf{b}\rangle$, where $\mathbf{a}$ is vector potential. Note however that one part of the time-scale used in Pouquet et al.'s calculation is based on Alfvén relaxation time. This assumption is suspect in view of current theoretical [1, 12, 13, 14, 15] and numerical results [16, 17, 18], which favour $k^{-5 / 3}$ (Kolmogorov's) energy spectrum over Kraichnan's $k^{-3 / 2}$ spectrum for MHD turbulence. In our present paper, the nonlinear time-scale is based on Kolmogorov's energy spectrum. The direct numerical simulation (DNS) of Pouquet and Patterson [19] yielded a similar result. The results of Brandenburg [20] discussed above are also obtained numerically. Frick and Sokoloff [21] studied the shell model of turbulence, and showed that magnetic helicity suppresses turbulent cascade.

In another development Dar et al. 22] numerically calculated various energy fluxes in two-dimensional MHD and showed that there is significant energy transfer from LS velocity field to LS magnetic field; they claimed that the above flux is one of the main contributor to the amplification of large-scale ME. Note that the ratio ME/KE grows in both helical and nonhelical MHD as well as in many decaying as well as forced simulations (see for example, 22, 23. and references therein). Hence, helicity is not a necessary requirement for the generation of magnetic energy. Regarding flux of magnetic helicity, Pouquet et al. [5] and Pouquet and Patterson [19] argue that it is in the inverse direction (from SS to LS). We find a similar magnetic helicity flux in our theoretical calculation.

In one of the recent theoretical development Kulsrud and Anderson 24] derived and solved the kinetic equation for the growth of galactic magnetic field. They argued that the dynamo time-scale is much large than the growth time-scale of turbulent modes. Hence, the buildup of SS turbulent ME dominates the slow growth of LS ME, thus making the mean dynamo theory invalid.

In paper I and the present paper, the energy fluxes of MHD turbulence are computed using perturbative field theory. The calculation is to first order in perturbation. Here the viscosity and resistivity were taken from renormalization calculation of Verma [14, 15], and the energy spectrum were taken to Kolmogorov-like $\left(k^{-5 / 3}\right)$. Kolmogorov's spectrum for MHD turbulence is supported by recent theoretical [11, 12, 13, 14, 15] and numerical results 16, 17, 18]. In paper I we show that in a kinetically forced nonhelical MHD, the energy transfer from LS velocity field to LS magnetic field is one of the dominant transfers. It is also shown in paper I that the above energy flux into LS magnetic modes is independent of the nature of LS forcing. In this paper we generalize the field-theoretic calculation of Verma 3 , 14] to helical MHD. As discussed in paper I, we assume that the turbulence is homogeneous and isotropic, and that the mean magnetic field is absent. The absence of mean magnetic field is a reasonable assumption for the initial stages of dynamo evolution. This assumption is to ensure that the turbulence is isotropic. In addition we take cross helicity $(\mathbf{u} \cdot \mathbf{b})$ to be zero to simplify the calculation. We examine the fluxes in the presence of magnetic and kinetic helicities. We also investigate the flux of magnetic helicity.

We have constructed a simple dynamo model using the theoretically-calculated energy flux into the large-scale magnetic field. In this model the ME grows exponentially in the initial stage, and the growth time-scale is of the order of eddy turnover time. It shows that dynamo action is possible in galactic dynamo.

The outline of the paper is as follows: in section 2, we carry out the perturbative calculation of renormalized viscosity and resistivity, as well as fluxes of energy and magnetic helicity. In section 3, we construct a dynamic galactic dynamo based on our energy flux results. In this section we also compare our results with the findings of earlier researchers. Section 4 contains conclusions.

\section{FIELD-THEORETIC CALCULATION OF HELICAL MAGNETOHYDRODYNAMICS}

The incompressible MHD equation in Fourier space is given by

$$
\begin{aligned}
\left(-i \omega+\nu k^{2}\right) u_{i}(\hat{k}) & =-\frac{i}{2} P_{i j m}^{+}(\mathbf{k}) \int d \hat{p}\left[u_{j}(\hat{p}) u_{m}(\hat{k}-\hat{p})-b_{j}(\hat{p}) b_{m}(\hat{k}-\hat{p})\right] \\
\left(-i \omega+\eta k^{2}\right) b_{i}(\hat{k}) & =-i P_{i j m}^{-}(\mathbf{k}) \int d \hat{p}\left[u_{j}(\hat{p}) b_{m}(\hat{k}-\hat{p})\right] \\
k_{i} u_{i}(\mathbf{k}) & =0 \\
k_{i} b_{i}(\mathbf{k}) & =0
\end{aligned}
$$

where $\mathbf{u}$ and $\mathbf{b}$ are the velocity and magnetic field fluctuations respectively, $\nu$ and $\eta$ are the viscosity and the resistivity respectively, and

$$
P_{i j m}^{+}(\mathbf{k}) \quad=\quad k_{j} P_{i m}(\mathbf{k})+k_{m} P_{i j}(\mathbf{k})
$$




$$
\begin{array}{rll}
P_{i m}(\mathbf{k}) & = & \delta_{i m}-\frac{k_{i} k_{m}}{k^{2}} ; \\
P_{i j m}^{-}(\mathbf{k}) & = & k_{j} \delta_{i m}-k_{m} \delta_{i j} ; \\
\hat{k}=(\mathbf{k}, \omega) ; & d \hat{p}=d \mathbf{p} d \omega /(2 \pi)^{4} .
\end{array}
$$

Note that we are working in three space dimension and also with zero mean magnetic field.

Some of the definitions regarding kinetic and magnetic helicities are in order. Throughout this paper, a denotes the vector potential $(\mathbf{b}=\nabla \times \mathbf{a})$, and $\boldsymbol{\Omega}$ denotes the vorticity $(\boldsymbol{\Omega}=\nabla \times \mathbf{u})$. The spectrum of helicity, $H_{M}(\mathbf{k})$, is defined using the equal-time correlation function $\left\langle a_{i}(\mathbf{k}, t) b_{j}(\mathbf{k}, t)\right\rangle$ (the angular brackets denote ensemble average),

$$
\left\langle a_{i}(\mathbf{k}, t) b_{j}\left(\mathbf{k}^{\prime}, t\right)\right\rangle \stackrel{\text { def }}{=} P_{i j}(\mathbf{k}) H_{M}(\mathbf{k})(2 \pi)^{3} \delta\left(\mathbf{k}+\mathbf{k}^{\prime}\right)
$$

The factor $P_{i j}(\mathbf{k})$ appears due to the constraints $\nabla \cdot \mathbf{a}=\nabla \cdot \mathbf{b}=0$. Using this correlation function we derive the following relationship:

$$
\begin{aligned}
H_{M} & =\frac{1}{2}\langle\mathbf{a}(\mathbf{x}) \cdot \mathbf{b}(\mathbf{x})\rangle \\
& =\frac{1}{2} \int \frac{d \mathbf{k}}{(2 \pi)^{3}} \frac{d \mathbf{k}^{\prime}}{(2 \pi)^{3}}\left\langle\mathbf{a}(\mathbf{k}) \cdot \mathbf{b}\left(\mathbf{k}^{\prime}\right)\right\rangle \\
& =\int \frac{d \mathbf{k}}{(2 \pi)^{3}} H_{M}(\mathbf{k})
\end{aligned}
$$

The one-dimensional magnetic helicity $H_{M}(k)$ is defined using

$$
\int H_{M}(k) d k=\int \frac{d \mathbf{k}}{(2 \pi)^{3}} H_{M}(\mathbf{k})
$$

Therefore,

$$
H_{M}(k)=\frac{4 \pi k^{2}}{(2 \pi)^{3}} H_{M}(\mathbf{k})
$$

Using $\nabla \times \mathbf{a}=\mathbf{b}$ we can easily derive that

$$
\mathbf{a}(\mathbf{k})=\frac{i}{k^{2}} \mathbf{k} \times \mathbf{b}(\mathbf{k})
$$

which leads to

$$
\left\langle b_{i}(\mathbf{k}) b_{j}\left(\mathbf{k}^{\prime}\right)\right\rangle=\left[P_{i j}(\mathbf{k}) C^{b b}(\mathbf{k})-i \epsilon_{i j l} k_{l} H_{M}(k)\right](2 \pi)^{3} \delta\left(\mathbf{k}+\mathbf{k}^{\prime}\right)
$$

where $C^{b b}$ is the $b-b$ correlation function.

A similar analysis for kinetic helicity shows that

$$
\begin{gathered}
\left\langle v_{i}(\mathbf{k}) \Omega_{j}\left(\mathbf{k}^{\prime}\right)\right\rangle \stackrel{\text { def }}{=} P_{i j}(\mathbf{k}) H_{K}(\mathbf{k}) \delta\left(\mathbf{k}+\mathbf{k}^{\prime}\right) \\
H_{K}=\frac{1}{2}\langle\mathbf{u} \cdot \boldsymbol{\Omega}\rangle=\int \frac{d \mathbf{k}}{(2 \pi)^{3}} H_{K}(\mathbf{k})
\end{gathered}
$$

and

$$
\left\langle u_{i}(\mathbf{k}) u_{j}\left(\mathbf{k}^{\prime}\right)\right\rangle=\left[P_{i j}(\mathbf{k}) C^{u u}(\mathbf{k})-i \epsilon_{i j l} k_{l} \frac{H_{K}(k)}{k^{2}}\right](2 \pi)^{3} \delta\left(\mathbf{k}+\mathbf{k}^{\prime}\right)
$$

where $C^{u u}$ is the $u-u$ correlation function.

An important point to note is that magnetic helicity is conserved in MHD when $\nu=\eta=0$. One of the consequences of this conservation law is the emergence of $k^{-1}$ spectrum at smaller wavenumbers 25]. The kinetic helicity is conserved in fluid turbulence, but not in MHD turbulence.

In the following subsection we will calculate the renormalized viscosity and resistivity for helical MHD. 


\section{A. Calculation of renormalized parameters}

Recently Verma [14, 15] has calculated the renormalized viscosity and resistivity for MHD turbulence in absence of kinetic and magnetic helicity. It will be shown below that the presence of both kinetic and magnetic helicities does not alter the renormalized viscosity and resistivity calculated for nonhelical MHD.

In the RG procedure the wavenumber range $\left(k_{N}, k_{0}\right)$ is divided logarithmically into $N$ shells. Then the elimination of the first shell $k^{>}=\left(k_{1}, k_{0}\right)$ is carried out and the modified MHD equation for $k^{<}=\left(k_{N}, k_{1}\right)$ is obtained. This process is continued for higher shells. The shell elimination is performed by ensemble averaging over $k^{>}$modes [14, 15, 26]. It is assumed that $u_{i}^{>}(\hat{k})$ and $b_{i}^{>}(\hat{k})$ have gaussian distributions with zero mean, while $u_{i}^{<}(\hat{k})$ and $b_{i}^{<}(\hat{k})$ are unaffected by the averaging process. In addition it is also assumed that

$$
\begin{aligned}
\left\langle u_{i}^{>}(\hat{p}) u_{j}^{>}(\hat{q})\right\rangle & =\left[P_{i j}(\mathbf{p}) C^{u u}(\hat{p})-i \epsilon_{i j l} \frac{p_{l}}{p^{2}} H_{K}(\hat{p})\right](2 \pi)^{4} \delta(\hat{p}+\hat{q}) \\
\left\langle b_{i}^{>}(\hat{p}) b_{j}^{>}(\hat{q})\right\rangle & =\left[P_{i j}(\mathbf{p}) C^{b b}(\hat{p})-i \epsilon_{i j l} p_{l} H_{M}(\hat{p})\right](2 \pi)^{4} \delta(\hat{p}+\hat{q})
\end{aligned}
$$

Note that $u-b$ correlation has been taken to be zero in our calculation.

We apply first-order perturbation theory to compute the renormalized parameters. After elimination of $n$ shells, we obtain the following equations for the renormalized viscosity $\nu_{(n)}$ and renormalized resistivity $\eta_{(n)}$ (for details refer to Verma [15]).

$$
\begin{aligned}
& \left(-i \omega+\nu_{(n)} k^{2}+\delta \nu_{(n)} k^{2}\right) u_{i}^{<(\hat{k})}=-\frac{i}{2} P_{i j m}^{+}(\mathbf{k}) \int d \hat{p}\left[u_{j}^{<}(\hat{p}) u_{m}^{<}(\hat{k}-\hat{p})\right. \\
& \left.-b_{j}^{<}(\hat{p}) b_{m}^{<}(\hat{k}-\hat{p})\right] \\
& \left(-i \omega+\eta_{(n)} k^{2}+\delta \eta_{(n)} k^{2}\right) b_{i}^{<}(\hat{k})=-i P_{i j m}^{-}(\mathbf{k}) \int d \hat{p}\left[u_{j}^{<}(\hat{p}) b_{m}^{<}(\hat{k}-\hat{p})\right]
\end{aligned}
$$

where

$$
\begin{gathered}
\delta \nu_{(n)}(k)=\frac{1}{2 k^{2}} \int_{\hat{p}+\hat{q}=\hat{k}}^{\Delta} \frac{d \mathbf{p}}{(2 \pi)^{3}}\left[\frac{S(k, p, q) C^{u u}(q)+S^{\prime}(k, p, q) H_{K}(q)}{\nu_{(n)}(p) p^{2}+\nu_{(n)}(q) q^{2}}\right. \\
\left.-\frac{S_{6}(k, p, q) C^{b b}(q)+S_{6}^{\prime}(k, p, q) H_{M}(q)}{\eta_{(n)}(p) p^{2}+\eta_{(n)}(q) q^{2}}\right] \\
\delta \eta_{(n)}(k)=\frac{1}{2 k^{2}} \int_{\hat{p}+\hat{q}=\hat{k}}^{\Delta} \frac{d \mathbf{p}}{(2 \pi)^{3}}\left[\begin{array}{c}
-\frac{S_{8}(k, p, q) C^{b b}(q)+S_{8}^{\prime}(k, p, q) H_{M}(q)}{\nu_{(n)}(p) p^{2}+\eta_{(n)}(q) q^{2}} \\
+\frac{S_{9}(k, p, q) C^{u u}(q)+S_{9}^{\prime}(k, p, q) H_{k}(q)}{\eta_{(n)}(p) p^{2}+\nu_{(n)}(q) q^{2}}
\end{array}\right]
\end{gathered}
$$

The quantities $S_{i}$ and $S_{i}^{\prime}$ are as follows:

$$
\begin{aligned}
S(k, p, q) & =P_{b j m}^{+}(k) P_{m a b}^{+}(p) P_{j a}(q)=2 k p\left(z^{3}+x y\right) \\
S_{6}(k, p, q) & =P_{a j m}^{+}(k) P_{m b a}^{-}(p) P_{j b}(q)=-2 k p z\left(1-y^{2}\right) \\
S_{8}(k, p, q) & =P_{i j m}^{-}(k) P_{j a b}^{+}(p) P_{m a}(q) P_{i b}(k)=S_{6}(p, k, q) \\
S_{9}(k, p, q) & =P_{i j m}^{-}(k) P_{m a b}^{-}(p) P_{j a}(q) P_{i b}(k)=2 k p(z+x y) \\
S^{\prime}(k, p, q) & =P_{b j m}^{+}(k) P_{m a b}^{+}(p) \epsilon_{j a l} q_{l}=0 \\
S_{6}^{\prime}(k, p, q) & =P_{a j m}^{+}(k) P_{m b a}^{-}(p) \epsilon_{j a l} q_{l}=0 \\
S_{8}^{\prime}(k, p, q) & =P_{i j m}^{-}(k) P_{j a b}^{+}(p) \epsilon_{m a l} q_{l} P_{i b}(k)=0 \\
S_{9}^{\prime}(k, p, q) & =P_{i j m}^{-}(k) P_{m a b}^{-}(p) \epsilon_{j a l} q_{l} P_{i b}(k)=0
\end{aligned}
$$

Since $\delta \nu$ and $\delta \eta$ are proper scalars and $H_{M, K}$ are pseudo scalars, $S_{i}^{\prime}(k, p, q)$ will be pseudo scalars. In addition, $S_{i}^{\prime}(k, p, q)$ are also linear in $k, p$ and $q$. This implies that $S_{i}^{\prime}(k, p, q)$ must be proportional to $\mathbf{q} \cdot(\mathbf{k} \times \mathbf{p})$, which will be zero because $\mathbf{k}=\mathbf{p}+\mathbf{q}$. Hence all $S_{i}^{\prime}(k, p, q)$ turn out to be zero, as a consequence the presence of helicities does not alter the already calculated $\delta(\nu, \eta)_{(n)}(k)$ by Verma [14, 15]. Zhou 27] arrived at a similar conclusion while calculating the renormalized viscosity for helical fluid turbulence. 
Verma 14, 15] obtained a self-consistent solution of the renormalized parameters using Kolmogorov's spectrum. Since the helicities do not alter the renormalized parameters, we arrive at the same formula for renormalized viscosity and resisitivity as Verma [14, 15], that is,

$$
(\nu, \eta)(k)= \begin{cases}\left(K^{u}\right)^{1 / 2} \Pi^{1 / 3} k^{-4 / 3}\left(\nu^{*}, \eta^{*}\right) & \text { for } k \geq k_{n} \\ \left(K^{u}\right)^{1 / 2} \Pi^{1 / 3} k_{n}^{-4 / 3}\left(\nu^{*}, \eta^{*}\right) & \text { for } k \leq k_{n}\end{cases}
$$

where $\Pi$ is the total energy flux, $K^{u}$ is the Kolmogorov's constant, and $\nu^{*}$ and $\eta^{*}$ are the renormalized parameters. The value of these renormalized parameters have been listed in [14, 15].

The present calculation has been carried out up to first order. The probability distribution of velocity is gaussian, while that of velocity difference is nongaussian. The nongaussian behaviour of velocity difference has significant effects specially on higher order structure functions, which are not properly accounted for by first order calculations. Yet, the first order calculation of renormalized viscosity yields results very close to those obtained in experiments and numerical simulations (see e.g., 26, 28]). For the above reason, we have stuck to the first-order field-theoretic calculation in the present paper.

In the next subsection we will calculate the energy and helicity fluxes using the field theoretic technique.

\section{B. Calculation of energy and helicity fluxes}

In paper I we have analytically calculated energy fluxes in the absence of magnetic and kinetic helicities. In this subsection we will generalize that calculation for helical MHD. Refer to paper I for the energy evolution equations and other basic formulas.

As discussed in paper I, the energy flux from inside of the $X$-sphere $(X<)$ to outside of the $Y$-sphere $(Y>)$ is

$$
\Pi_{Y>}^{X<}\left(k_{0}\right)=\frac{1}{(2 \pi)^{3} \delta\left(\mathbf{k}^{\prime}+\mathbf{p}+\mathbf{q}\right)} \int_{k^{\prime}>k_{0}} \frac{d \mathbf{k}^{\prime}}{(2 \pi)^{3}} \int_{p<k_{0}} \frac{d \mathbf{p}}{(2 \pi)^{3}}\left\langle S^{Y X}\left(\mathbf{k}^{\prime}|\mathbf{p}| \mathbf{q}\right)\right\rangle
$$

where $X$ and $Y$ stand for $u$ or $b$, and $S\left(\mathbf{k}^{\prime}|\mathbf{p}| \mathbf{q}\right)$ is energy transfer from mode $\mathbf{p}$ of $X$ field to mode $\mathbf{k}$ of $Y$ field, with mode $\mathbf{q}$ acting as a mediator. The detailed expressions for $\left\langle S^{Y X}\left(\mathbf{k}^{\prime}|\mathbf{p}| \mathbf{q}\right)\right\rangle$ are given in Paper I.

We calculate the above fluxes analytically to the leading order in perturbation series using the same procedure as in paper I. Some additional terms appear in $\left\langle S\left(k^{\prime}|p| q\right)\right\rangle$ due to the presence of helicity. The detailed expressions are given in Appendix A.

A formula for the magnetic helicity flux can be derived in a similar manner. From Eqs. (2 3) we can easily obtain the equation for the evolution of magnetic helicity, which is

$$
\begin{aligned}
\frac{\partial H_{M}(\mathbf{k})}{\partial t} & =\frac{1}{2(2 \pi)^{3} \delta\left(\mathbf{k}+\mathbf{k}^{\prime}\right)} \Re\left[\mathbf{b}^{*}(\mathbf{k}) \cdot \frac{\partial \mathbf{a}\left(\mathbf{k}^{\prime}\right)}{\partial t}+\mathbf{a}^{*}(\mathbf{k}) \cdot \frac{\partial \mathbf{b}\left(\mathbf{k}^{\prime}\right)}{\partial t}\right] \\
& =\frac{1}{2(2 \pi)^{3} \delta\left(\mathbf{k}+\mathbf{k}^{\prime}\right)}\left[S^{H_{M}}\left(\mathbf{k}^{\prime}|\mathbf{p}| \mathbf{q}\right)+S^{H_{M}}\left(\mathbf{k}^{\prime}|\mathbf{q}| \mathbf{p}\right)\right]
\end{aligned}
$$

where

$$
\begin{aligned}
S^{H_{M}}\left(\mathbf{k}^{\prime}|\mathbf{p}| \mathbf{q}\right)= & \frac{1}{4} \Re\left[\mathbf{b}\left(\mathbf{k}^{\prime}\right) \cdot(\mathbf{v}(\mathbf{p}) \times \mathbf{b}(\mathbf{q}))\right] \\
& +\frac{1}{4} \Im\left[\mathbf{k}^{\prime} \cdot \mathbf{b}(\mathbf{q}) \mathbf{a}\left(\mathbf{k}^{\prime}\right) \cdot \mathbf{u}(\mathbf{p})-\mathbf{k}^{\prime} \cdot \mathbf{u}(\mathbf{q}) \mathbf{a}\left(\mathbf{k}^{\prime}\right) \cdot \mathbf{b}(\mathbf{p})\right]
\end{aligned}
$$

Here $\Re()$ and $\Im()$ stand for real and imaginary part of the arguments, respectively. The quantity $S^{H_{M}}\left(\mathbf{k}^{\prime}|\mathbf{q}| \mathbf{p}\right)$ can be obtained from the above expression by interchanging $\mathbf{p}$ and $\mathbf{q}$. After some algebraic manipulation it can be shown that

$$
\begin{aligned}
& S^{H_{M}}\left(\mathbf{k}^{\prime}|\mathbf{p}| \mathbf{q}\right)+S^{H_{M}}\left(\mathbf{k}^{\prime}|\mathbf{q}| \mathbf{p}\right)+S^{H_{M}}\left(\mathbf{p}\left|\mathbf{k}^{\prime}\right| \mathbf{q}\right) \\
+ & S^{H_{M}}\left(\mathbf{p}|\mathbf{q}| \mathbf{k}^{\prime}\right)+S^{H_{M}}\left(\mathbf{q}\left|\mathbf{k}^{\prime}\right| \mathbf{p}\right)+S^{H_{M}}\left(\mathbf{q}|\mathbf{p}| \mathbf{k}^{\prime}\right)=0
\end{aligned}
$$

It shows that the "detailed conservation of magnetic helicity" holds in a triad interaction (when $\nu=\eta=0$ ) 29].

From the above, the transfer rate of magnetic helicity from a wavenumber sphere of radius $k_{0}$ is

$$
\Pi_{H_{M}}\left(k_{0}\right)=\frac{1}{(2 \pi)^{3} \delta\left(\mathbf{k}^{\prime}+\mathbf{p}+\mathbf{q}\right)} \int_{k^{\prime}>k_{0}} \frac{d \mathbf{k}^{\prime}}{(2 \pi)^{3}} \int_{p<k_{0}} \frac{d \mathbf{p}}{(2 \pi)^{3}}\left\langle S^{H_{M}}\left(\mathbf{k}^{\prime}|\mathbf{p}| \mathbf{q}\right)\right\rangle
$$


We again compute $\left\langle S^{H_{M}}\left(\mathbf{k}^{\prime}|\mathbf{p}| \mathbf{q}\right)\right\rangle$ to first order in perturbation. The detailed expressions are given in Appendix B.

The expressions in the Appendices involve Green's functions and correlation functions. The expressions for these functions are taken from self-consistent calculations (see e.g., Verma 15]). For $G\left(k, t-t^{\prime}\right)$ of the formulas A1 A4 B3, we substitute

$$
G^{(u u, b b)}\left(k, t-t^{\prime}\right)=\theta\left(t-t^{\prime}\right) \exp \left[-(\nu(k), \eta(k)) k^{2}\left(t-t^{\prime}\right)\right]
$$

where $(\nu(k), \eta(k))$ are given by Eq. (33), and $\theta\left(t-t^{\prime}\right)$ is the step function. We assume the relaxation time-scale for $C^{u u}\left(k, t, t^{\prime}\right)$ and $H_{K}\left(k, t, t^{\prime}\right)$ to be $\left(\nu(k) k^{2}\right)^{-1}$, while that of $C^{b b}\left(k, t, t^{\prime}\right)$ and $H_{M}\left(k, t, t^{\prime}\right)$ to be $\left(\eta(k) k^{2}\right)^{-1}$. The spectrum $C^{(u u, b b)}(k, t, t)$ are written in terms of one-dimensional energy spectra $E^{(u, b)}$ as

$$
C^{(u u, b b)}(k, t, t)=\frac{(2 \pi)^{3}}{4 \pi k^{2}} E^{(u, b)}
$$

In presence of magnetic helicity, the calculations based on absolute equilibrium theories suggest that the energy cascades forward, and the magnetic helicity cascades backward [25]. In this paper we have not considered the inverse cascade region of magnetic helicity. We take Kolmogorov's spectrum for energy based on recent numerical simulations [16, 17, 18] and theoretical calculations [11, 12, 13, 15] (ignoring the intermittency corrections). Hence, the spectrum of $E^{(u, b)}$ can be taken as

$$
\begin{aligned}
E^{u}(k) & =K^{u} \Pi^{2 / 3} k^{-5 / 3} \\
E^{b}(k) & =E^{u} / r_{A}
\end{aligned}
$$

where $\Pi$ is total energy flux.

The helicities are written in terms of energy spectra as

$$
\begin{aligned}
& H_{K}(k)=r_{K} k E^{u}(k) \\
& H_{M}(k)=r_{M} \frac{E^{b}(k)}{k}
\end{aligned}
$$

We are calculating energy fluxes for the inertial-range wavenumbers where the same powerlaw is valid for all energy spectrum. Therefore, the ratios $r_{A}, r_{M}$, and $r_{K}$ can be treated as constants.

We substitute the above forms for the correlation and Green's functions [Eqs. (40.43)] in the expressions for $\left\langle S^{Y X}\left(k^{\prime}|p| q\right)\right\rangle$ and $\left\langle S^{H_{M}}\left(k^{\prime}|p| q\right)\right\rangle$ given in the Appendices. These $S$ 's are substituted in the flux formulas (Eqs. 34] 39). We make the following change of variable:

$$
k=\frac{k_{0}}{u} ; p=\frac{k_{0}}{u} v ; q=\frac{k_{0}}{u} w
$$

These operations yield the following nondimensional form of the equation in the $-5 / 3$ region (for details, refer to [3] ).

$$
\begin{aligned}
& \frac{\Pi_{Y>}^{X<}\left(k_{0}\right)}{\Pi\left(k_{0}\right)}=\left(K^{u}\right)^{3 / 2}\left[\frac{1}{2} \int_{0}^{1} d v \ln (1 / v) \int_{1-v}^{1+v} d w(v w) \sin \alpha F_{Y>}^{X<}\right] \\
& \frac{\Pi_{H_{M}}\left(k_{0}\right)}{\Pi\left(k_{0}\right)}=\frac{1}{k_{0}}\left(K^{u}\right)^{3 / 2}\left[\frac{1}{2} \int_{0}^{1} d v(1-v) \int_{1-v}^{1+v} d w(v w) \sin \alpha F_{H_{M}}\right]
\end{aligned}
$$

where the integrands $\left(F_{Y>}^{X<}, F_{H_{M}}\right)$ are function of $v, w, \nu^{*}, \eta^{*}, r_{A}, r_{K}$ and $r_{M}[\underline{3}]$.

We compute the term in the square brackets, $I_{Y>}^{X<}$, using the similar procedure as that of Verma [3]. The flux ratios $\Pi_{Y>}^{X<} / \Pi$ can be written in terms of integrals $I_{Y>}^{X<}$, which have been computed numerically. Table $\llbracket$ contains their values for $r_{A}=1$ and $r_{A}=5000$. The constant $K^{u}$ is calculated using the fact that the total energy flux $\Pi$ is sum of all $\Pi_{Y>}^{X}<$. For parameters $\left(r_{A}=5000, r_{K}=0.1, r_{M}=-0.1\right), K^{u}=1.53$, while for $\left(r_{A}=1, r_{K}=0.1, r_{M}=-0.1\right)$, $K^{u}=0.78$. After this the energy flux ratios $\Pi_{Y>}^{X<} / \Pi$ can be calculated. These ratios for some of the specific values of $r_{A}, r_{K}$ and $r_{M}$ are listed in Table [I] The first and second terms of $\Pi_{Y}^{X} / \Pi$ entries are nonhelical and helical components respectively.

An observation of the results shows some interesting patterns. The energy flux can be split into two parts: helical (dependent on $r_{K}$ and/or $r_{M}$ ) and nonhelical (independent of helicity). The nonhelical part of all the fluxes except $\Pi_{u>}^{b<}\left(\Pi_{u>\text { nonhelical }}^{b<}<0\right.$ for $\left.r_{A}>1\right)$ is always positive. As a consequence, in nonhelical channel, ME cascades from LS to SS. Also, since $\Pi_{b<}^{u<}>0$, LS kinetic energy feeds the LS magnetic energy. The fluxes of nonhelical MHD has been discussed in great detail in paper I. 
The sign of $\Pi_{u>h e l i c a l}^{u<}$ is always negative, i.e., kinetic helicity reduces the KE flux. But the sign of helical component of other energy fluxes depends quite crucially on the sign of helicities. From the entries of Table $\square$ we see that

$$
\Pi_{(b>, u>) h e l i c a l}^{b<}=-a r_{M}^{2}+b r_{M} r_{K}
$$

where $a$ and $b$ are positive constants. If $r_{M} r_{K}<0$, the energy flux to LS magnetic field due to both the terms in the right-hand-side of the above equation is positive. Earlier EDQNM [5] and numerical simulations [20] with forcing of KE and $H_{K}$ typically have $r_{K} r_{M}<0$. Hence, we can claim that helicity typically induces an inverse energy cascade via $\Pi_{b>}^{b<}$ and $\Pi_{u>}^{b<}$. These fluxes will enhance the large-scale magnetic field.

From the entries of Table large-scale magnetic field is less than the forward nonhelical energy flux $\Pi_{b>}^{\underline{i}}$. While for helical MHD $\left(r_{K}, r_{M} \rightarrow 1\right)$, the inverse helical cascade dominates the nonhelical magnetic-to-magnetic energy cascade.

The flux ratio $\Pi_{H_{M}} / \Pi$ can be written in terms the integrals of Eqs. (4848) using the same procedure as done for energy flux ratios. The numerical values of the integrals are shown in Tables 1 and 2. Clearly,

$$
\Pi_{H_{M}}=-d r_{M}+e r_{K}
$$

where $d$ and $e$ are positive constants. Note however that contribution of $H_{M}$ dominates that of $H_{K}$. Clearly the sign of $\Pi_{H_{M}}$ is the same as that of $H_{K}$ but negative of $H_{M}$. From the above equation we observe that positive $H_{M}$ yields a negative contribution to $\Pi_{H_{M}}$. Hence, for positive $H_{M}$, the magnetic helicity cascade is backward. This result is in agreement with Frisch et al.'s 25] argument in which they predict an inverse cascade of magnetic helicity. Our theoretical result on inverse cascade of $H_{M}$ is also in agreement with the results derived using EDQNM calculation [5] and numerical simulations [19].

When we force the system with positive kinetic helicity $\left(r_{K}>0\right)$, Eq. (50) indicates a forward cascade of magnetic energy. This effect could be the reason for the observe production of positive magnetic helicity at small scale by Brandenburg [20] Because of magnetic helicity conservation, he also finds generation of negative magnetic helicity at large-scales. Now, positive kinetic helicity and negative magnetic helicity at large-scales may yield an inverse cascade of magnetic energy (see Eq. 49). This could be the crude reason for the growth of magnetic energy in the simulations of Brandenburg [20].

In paper I we calculated $\Pi_{b<}^{u<}$ for nonhelical MHD using steady-state condition.

$$
\Pi_{b<}^{u<}=\Pi_{b>n o n h e l i c a l}^{b<}+\Pi_{u>\text { nonhelical }}^{b<} .
$$

The above calculation for helical MHD is not straight forward because magnetic energy at large-scale could increase with time, and steady state may not be achievable for all possible parameters of helical MHD. Brandenburg [20] observes the dynamic evolution of large-scale magnetic energy in his simulations. To simplify the calculation, we assume steady-state condition for helical MHD as well, and calculate various parameters. For some set of highly helical MHD, we get negative energy flux.

In the following section, we will construct a dynamic dynamo model for galaxies using our flux results.

\section{DYNAMO VIA ENERGY AND MAGNETIC HELICITY FLUXES}

In the above calculation we have assumed that the turbulence is homogeneous, isotropic, and steady. The assumption of homogeneity and isotropy can be assumed to hold in galaxies in the early phases of evolution before large structures appear. The assumption that the mean magnetic field of galaxy is rather small is valid in the beginning of the galactic evolution. Therefore, we apply the flux obtained from our calculations to estimate the growth of magnetic energy in galaxies.

During the early phase of galactic evolution, only the large-scales (LS) contain the kinetic and magnetic energies. The fields at these scales interact with each other, but the small-scale spectrum is far from steady (not enough time). The interactions of LS velocity field and the LS seed magnetic field increase the LS seed magnetic field $E^{b}(t)$ till the steady-state is reached. In absence of helicity, the source of energy for the large-scale magnetic field is $\Pi_{b<}^{u<}[$ Eq. (51)]. When helicity is present, there are several other sources as discussed in section 2 of this paper. Since the forcing of helicities is effective at LS, it is reasonable to assume that the helical part of $\Pi_{b<}^{b>}$ and $\Pi_{b<}^{u>}$ will also aid to the increase in LS ME. Hence,

$$
\frac{d E^{b}(t)}{d t}=\Pi_{b<}^{u<}+\Pi_{b<\text { helical }}^{b>}+\Pi_{b<\text { helical }}^{u>}
$$


We assume a quasi-steady approximation for the early evolution of magnetic field. In many quasi-steady situations (slowly decaying or growing), steady-state results are usually applied. This approximation works very well for many practical problems. We make this assumption in this paper, and substitute the theoretically calculated energy fluxes calculated in Section II of the paper to the above equation.

Since the ME starts with a small value (large $r_{A}$ limit), all the fluxes appearing in Eq. (52) are proportional to $r_{A}^{-1}$ [cf. Eqs. (A2A4 ], i.e.,

$$
\Pi_{b<}^{u<}+\Pi_{b<\text { helical }}^{b>}+\Pi_{b<h e l i c a l}^{u>}=c \Pi \frac{E^{b}}{E^{u}}
$$

where $E^{u}$ is the LS KE, and $c$ is the constant of proportionality, which depends on the values of helicities. Both Kinetic and magnetic helicities are difficult to ascertain for a galaxy due to lack of observations. We take $r_{M}$ and $r_{K}$ to be of the order of 0.1, with $r_{M}$ being negative. The choice of negative $r_{M}$ is motivated by the results of EDQNM calculation [5] and numerical simulations [20]. With this value of $r_{M}$ and $r_{K}, c \approx 0.84$ for $E(k) \propto k^{-5 / 3}$ regime, and $c \approx 1.3$ for $E(k) \propto k^{-1}$ regime. Since both the values of constant $c$ is approximately equal and close to 1.0 , we take $c=1.0$ for our calculation. Hence,

$$
\frac{1}{\Pi} \frac{d E^{b}}{d t} \approx \frac{E^{b}}{E^{u}}
$$

Using $E^{u}=K^{u} \Pi^{2 / 3} L^{2 / 3}$, where $L$ is the large length-scale of the system, we obtain

$$
\frac{1}{\sqrt{E^{u}} E^{b}} \frac{d E^{b}}{d t} \approx \frac{1}{L\left(K^{u}\right)^{3 / 2}}
$$

We assume that $E^{u}$ does not change appreciably in the early phase. Therefore,

$$
E^{b}(t) \approx E^{b}(0) \exp \left(\frac{\sqrt{E^{u}}}{L\left(K^{u}\right)^{3 / 2}} t\right)
$$

Hence, the ME grows exponentially in the early periods, and the time-scale of growth is of the order of $L\left(K^{u}\right)^{3 / 2} / \sqrt{E^{u}}$, which is the eddy turnover time [3]. Taking $L \approx 10^{17} \mathrm{~km}$ and $\sqrt{E^{u}} \approx 10 \mathrm{~km} / \mathrm{sec}$, we obtain the growth time-scale to be $10^{16}$ sec or $3 \times 10^{8}$ years, which is in the expected range [24]. Hence, we have constructed a nonlinear and dynamically consistent galactic dynamo based on the energy fluxes. In this model the ME grows exponentially, and the growth time-scale is reasonable 24].

The helical and nonhelical contribution to the fluxes for $r_{A}=5000, r_{K}=0.1, r_{M}=-0.1$ is shown in Table The flux ratios shown in the table do not change appreciably as long as $r_{A}>100$ or so. The three fluxes responsible for the growth of LS ME are $\Pi_{b<}^{u<} / \Pi \approx 2.6 \times 10^{-4}$ (nonhelical), $\Pi_{b>\text { helical }}^{b<} / \Pi \approx-4.1 \times 10^{-5}$, and $\Pi_{u>\text { helical }}^{b<} / \Pi \approx-4.0 \times 10^{-5}$. The ratio of nonhelical to helical contribution is $2.6 / 0.81 \approx 3.2$. Hence, the nonhelical contribution is significant, if not more, than the contribution from the helical part for the LS ME amplification. Note that in the earlier papers on dynamo, the helical part is strongly emphasized.

Kulsrad and Anderson (KA) 24] performed an important mean field dynamo calculation of galactic dynamo for large Prandtl numbers. Some of the salient features are follows. In KA's kinematic dynamo calculation the growth rate of $\mathrm{ME}$ is $\gamma=(1 / 3) \int k^{2} U(\mathbf{k}) d \mathbf{k}$ where

$$
U(\mathbf{k})=\frac{(\Pi)^{2 / 3} k^{-5 / 3}}{4 \pi k^{3} u_{k}} \approx(\Pi)^{1 / 3} k^{-13 / 3}
$$

KA estimate $\gamma \approx k_{\max }^{2 / 3}(\Pi)^{1 / 3} \approx 10^{-4} y r^{-1}$. From this result KA conclude that the kinematic theory predicts a extremely rapid growth of SS ME. The SS noisy magnetic field thus generated will dominate the mean magnetic field that grows at a considerably slower rate (dynamo growth time $\approx 3 \times 10^{8} \mathrm{yr}$ ). Therefore, it is claimed that the kinematic assumption of the mean dynamo theory is invalid, and it is difficult to build up galactic magnetic field from a very weak seed field using dynamo action. KA's estimate of growth time-scale is equal to the eddy turnover time of smallest eddies $\left(k_{\max }^{-1}\right)$. Hence, as pointed out by KA, kinematic assumption is invalid for galactic dynamo, and one has to resort to a dynamical model. Brandenburg's numerical results [20] are not quite consistent with KA's results. For example, Brandenburg finds (1) growth of magnetic energy even for large magnetic Prandtl number; (2) the growth time-scale for magnetic energy is of the order of $L^{2} / \eta$, where $L$ is the large length-scale, and $\eta$ is resistivity.

Our results are valid for Prandtl number close to 1 . Therefore, they can not be compared with KA's calculations. It is interesting to see however that our crude estimate of time scale is one eddy turn-over time. To get a better picture, we need to construct a more solid model. 
In our model the magnetic energy growth is due to the fluxes $\Pi_{b<}^{u<}+\Pi_{b<\text { helical }}^{b>}+\Pi_{b<\text { helical }}^{u>}$. In nonhelical MHD, only $\Pi_{b<}^{u<}$ is effective, while in helical MHD both kinetic and magnetic helicities play an important role in the growth of ME. In the current kinematic models of planetary magnetism [1], magnetic field is generated by kinetic helicity, for which planetary rotation (spin) plays an important role. These models appear to work for all the planets except Mercury, which rotates far too slowly. We conjecture that $\Pi_{b<}^{u<}$ (independent of helicities) probably plays an important role in the generation of magnetic field of Mercury.

In helical MHD, the helical contribution to the magnetic energy growth goes as [see Eq. [49]]

$$
\frac{d E^{b}}{d t}=a r_{M}^{2}-b r_{M} r_{K}
$$

where $a$ and $b$ are positive constants. The term $a r_{M}^{2}$ is always positive independent of the sign of $H_{M}$, but $-b r_{M} r_{K}$ is positive only when $H_{M}$ and $H_{K}$ are of the opposite sign. In numerical simulation of Brandenburg [20] and EDQNM calculation of Pouquet et al. [5], $H_{M} H_{K}<0$ for small $k$, and $H_{M} H_{K}>0$ for large $k$. Hence, $-b r_{M} r_{K}$ term is positive for small $k$, resulting in positive $d E^{b} / d t$. Let us compare the above result with the dynamical dynamo of Pouquet et al. [5], Field et al. 7], and Cho [8].

The kinematic dynamo predicts that the growth parameter $\alpha$ is proportional to $H_{K}$, i.e, $\alpha=\alpha_{u} \propto\langle\mathbf{u} \cdot \nabla \times \mathbf{u}\rangle$. The kinetic model was generalized by Pouquet et al. [5], Field et al. [7], and Cho [8]. In absence of a mean magnetic field they find that

$$
\alpha \approx \alpha_{u}+\alpha_{b}=\ldots\langle\mathbf{u} \cdot \nabla \times \mathbf{u}\rangle-\ldots\langle\mathbf{b} \cdot \nabla \times \mathbf{b}\rangle,
$$

where ... denotes certain time scales (which are always positive). It implies that $\alpha$ gets positive contribution from both the terms when $H_{M}$ and $H_{K}$ are of opposite signs. This result is consistent with Eq. (58). The direct numerical simulation of Pouquet and Patterson [19] indicate that $H_{M}$ enhances the growth rate of ME considerably, but that is not the case with $H_{K}$ alone. This numerical result is somewhat inconsistent with results of Pouquet et al. and others [5] (Eq. (59)), but it fits better our formula (58) $\left(d E^{b} / d t=0\right.$ if $\left.r_{M}=0\right)$. Hence, our formula (58) probably is a better model for the dynamically consistent dynamo.

In the following section we will summarize our results.

\section{CONCLUSIONS}

In this paper we have applied first-order perturbative field theory to calculate the renormalized viscosity, renormalized resistivity, and various cascade rates for helical MHD. We find that the renormalized viscosity and resistivity are unaffected on introduction of both kinetic and magnetic helicities. Our result is consistent with Zhou's calculation 27] for helical fluid turbulence.

We find that the energy cascade rates get significantly altered by helicity. Since magnetic helicity is a conserved quantity in MHD, Frisch [25] had argued for $k^{-1}$ energy spectra at small wavenumbers. However, in this paper we calculate energy fluxes in the Kolmogorov's inertial range, where we find direct energy cascades. The fluxes are shown in Table The main results of our calculation are as follows:

1. The magnetic energy flux has two components: (a) the nonhelical part which is always positive, (b) the helical part which is negative (assuming $H_{M} H_{K}<0$ ). The inverse cascade resulting due to helicities is consistent with the results of Pouquet et al. [5], Brandenburg [20], and others.

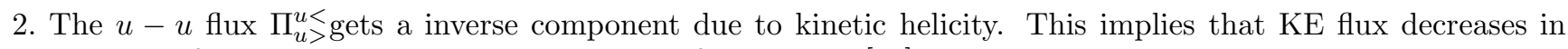
presence of $H_{K}$, a result consistent with that of Kraichnan [30].

3. The growth of large-scale magnetic field in the initial stage of evolution results from $\Pi_{b<}^{u<}+\Pi_{b<\text { helical }}^{b>}+\Pi_{b<\text { helical }}^{u>}$. In this paper we have computed the relative magnitudes of all three contributions, and find that all of them to be comparable, although $\Pi_{b<}^{u<}$ is somewhat higher. Pouquet et al. [5], Pouquet and Patterson [19], Brandenburg [20], and many others highlight $\Pi_{b<\text { helical }}^{b>}$ transfer, and generally do not consider $\Pi_{b<}^{u<}$ and $\Pi_{b<\text { helical }}^{u>}$ fluxes.

4. Regarding positive $H_{M}$, the flux of magnetic helicity $\Pi_{H_{M}}$ is backward.

Most of the earlier papers (e.g. Pouquet et al. [5]) assume Alfvén time-scale to be the dominant time-scale for MHD turbulence. We have taken nonlinear time-scale (based on Kolmogorov's spectrum) to be the relevant time-scale based on recent numerical [16, 17, 18] and theoretical [11, 12, 13, 14, 15] work. 
Using the flux results we have constructed a simple nonlinear and dynamically consistent galactic dynamo. Our model shows an exponential growth of magnetic energy in the early phase (much before saturation). The growth time-scale is of the order of $3 \times 10^{8}$ years, which is consistent with the current estimate [31]. In our paper we have discussed the growth of magnetic field at scale comparable to forcing scales. In real dynamo, the magnetic field at even larger scales also grow. This growth may be due to inverse cascade of magnetic energy. This problem is beyond the scope of our paper.

Some of the results presented here are general, and they are expected to hold in solar and planetary dynamo. For example, we find that LS velocity field supply energy to LS seed magnetic field. This is one of the sources of dynamo. Hence, if we solve first few MHD modes in spherical coordinate with kinetic forcing, it may be possible to capture some of the salient features of solar and planetary dynamo.

In summary, the energy flux studies of helical MHD provide us with many important insights into the problem of magnetic energy growth. Its application to galactic dynamo yields very interesting results. A generalization of the formalism presented here to spherical geometry may provide us with insights into the magnetic field generation in the Sun and Earth.

\section{Acknowledgments}

The author thanks G. Dar, V. Eswaran, A. Brandenburg, D. Narsimhan, and R. K. Varma for discussions. He also thanks Mustansir Barma (TIFR, Mumbai) and Krishna Kumar (ISI, Calcutta) for useful suggestions and kind hospitality during his stay in their institutes on his sabbatical leave. This work was supported in part by Department of Science and Technology, India.

\section{APPENDIX A: CALCULATION OF $\left\langle S^{Y X}\left(\mathbf{k}^{\prime}|\mathbf{p}| \mathbf{q}\right)\right\rangle$}

The expressions for $\left\langle S^{Y X}\right\rangle$ for helical MHD are

$$
\begin{aligned}
& \left\langle S^{u u}\left(k^{\prime}|p| q\right)\right\rangle=\int_{-\infty}^{t} d t^{\prime}(2 \pi)^{3}\left[T_{1}(k, p, q) G^{u u}\left(k, t-t^{\prime}\right) C^{u}\left(p, t, t^{\prime}\right) C^{u}\left(q, t, t^{\prime}\right)\right. \\
& +T_{1}^{\prime}(k, p, q) G^{u u}\left(k, t-t^{\prime}\right) \frac{H_{K}\left(p, t, t^{\prime}\right)}{p^{2}} \frac{H_{K}\left(q, t, t^{\prime}\right)}{q^{2}} \\
& +T_{5}(k, p, q) G^{u u}\left(p, t-t^{\prime}\right) C^{u}\left(k, t, t^{\prime}\right) C^{u}\left(q, t, t^{\prime}\right) \\
& +T_{5}^{\prime}(k, p, q) G^{u u}\left(p, t-t^{\prime}\right) \frac{H_{K}\left(k, t, t^{\prime}\right)}{k^{2}} \frac{H_{K}\left(q, t, t^{\prime}\right)}{q^{2}} \\
& +T_{9}(k, p, q) G^{u u}\left(q, t-t^{\prime}\right) C^{u}\left(k, t, t^{\prime}\right) C^{u}\left(p, t, t^{\prime}\right) \\
& \left.+T_{9}^{\prime}(k, p, q) G^{u u}\left(q, t-t^{\prime}\right) \frac{H_{K}\left(k, t, t^{\prime}\right)}{k^{2}} \frac{H_{K}\left(p, t, t^{\prime}\right)}{p^{2}}\right] \\
& -\left\langle S^{u b}\left(k^{\prime}|p| q\right)\right\rangle=\int_{-\infty}^{t} d t^{\prime}(2 \pi)^{3}\left[T_{2}(k, p, q) G^{u u}\left(k, t-t^{\prime}\right) C^{b}\left(p, t, t^{\prime}\right) C^{b}\left(q, t, t^{\prime}\right)\right. \\
& +T_{2}^{\prime}(k, p, q) G^{u u}\left(k, t-t^{\prime}\right) H_{M}\left(p, t, t^{\prime}\right) H_{M}\left(q, t, t^{\prime}\right) \\
& +T_{7}(k, p, q) G^{b b}\left(p, t-t^{\prime}\right) C^{u}\left(k, t, t^{\prime}\right) C^{b}\left(q, t, t^{\prime}\right) \\
& +T_{7}^{\prime}(k, p, q) G^{b b}\left(p, t-t^{\prime}\right) \frac{H_{K}\left(k, t, t^{\prime}\right)}{k^{2}} H_{M}\left(q, t, t^{\prime}\right) \\
& +T_{11}(k, p, q) G^{u u}\left(q, t-t^{\prime}\right) C^{u}\left(k, t, t^{\prime}\right) C^{b}\left(p, t, t^{\prime}\right) \\
& \left.+T_{11}^{\prime}(k, p, q) G^{u u}\left(q, t-t^{\prime}\right) \frac{H_{K}\left(k, t, t^{\prime}\right)}{k^{2}} H_{M}\left(p, t, t^{\prime}\right)\right] \\
& -\left\langle S^{b u}\left(k^{\prime}|p| q\right)\right\rangle=\int_{-\infty}^{t} d t^{\prime}(2 \pi)^{3}\left[T_{3}(k, p, q) G^{b b}\left(k, t-t^{\prime}\right) C^{u}\left(p, t, t^{\prime}\right) C^{b}\left(q, t, t^{\prime}\right)\right. \\
& +T_{3}^{\prime}(k, p, q) G^{b b}\left(k, t-t^{\prime}\right) \frac{H_{K}\left(p, t, t^{\prime}\right)}{p^{2}} H_{M}\left(q, t, t^{\prime}\right) \\
& +T_{6}(k, p, q) G^{u u}\left(p, t-t^{\prime}\right) C^{b}\left(k, t, t^{\prime}\right) C^{b}\left(q, t, t^{\prime}\right) \\
& +T_{6}^{\prime}(k, p, q) G^{u u}\left(p, t-t^{\prime}\right) H_{M}\left(k, t, t^{\prime}\right) H_{M}\left(q, t, t^{\prime}\right)
\end{aligned}
$$




$$
\begin{aligned}
& +T_{12}(k, p, q) G^{b b}\left(q, t-t^{\prime}\right) C^{b}\left(k, t, t^{\prime}\right) C^{u}\left(p, t, t^{\prime}\right) \\
& \left.+T_{12}^{\prime}(k, p, q) G^{b b}\left(q, t-t^{\prime}\right) H_{M}\left(k, t, t^{\prime}\right) \frac{H_{K}\left(p, t, t^{\prime}\right)}{p^{2}}\right] \\
\left\langle S^{b b}\left(k^{\prime}|p| q\right)\right\rangle=\int_{-\infty}^{t} d & t^{\prime}(2 \pi)^{3}\left[T_{4}(k, p, q) G^{b b}\left(k, t-t^{\prime}\right) C^{b}\left(p, t, t^{\prime}\right) C^{u}\left(q, t, t^{\prime}\right)\right. \\
& +T_{4}^{\prime}(k, p, q) G^{b b}\left(k, t-t^{\prime}\right) H_{M}\left(p, t, t^{\prime}\right) \frac{H_{K}\left(q, t, t^{\prime}\right)}{q^{2}} \\
& +T_{8}(k, p, q) G^{b b}\left(p, t-t^{\prime}\right) C^{b}\left(k, t, t^{\prime}\right) C^{u}\left(q, t, t^{\prime}\right) \\
& +T_{8}^{\prime}(k, p, q) G^{b b}\left(p, t-t^{\prime}\right) H_{M}\left(k, t, t^{\prime}\right) \frac{H_{K}\left(q, t, t^{\prime}\right)}{q^{2}} \\
& +T_{10}(k, p, q) G^{u u}\left(q, t-t^{\prime}\right) C^{b}\left(k, t, t^{\prime}\right) C^{b}\left(p, t, t^{\prime}\right) \\
& \left.+T_{10}^{\prime}(k, p, q) G^{u u}\left(q, t-t^{\prime}\right) H_{M}\left(k, t, t^{\prime}\right) H_{M}\left(p, t, t^{\prime}\right)\right]
\end{aligned}
$$

where $T_{i}(k, p, q)$ are given in paper I. To obtain $T_{i}^{\prime}(k, p, q)$ (helical part) we replace all the second rank tensors of the type $P_{j a}(k)$ by $\epsilon_{j a l} k_{l}$.

\section{APPENDIX B: CALCULATION OF $\left\langle S^{H_{M}}\left(\mathbf{k}^{\prime}|\mathbf{p}| \mathbf{q}\right)\right\rangle$}

The quantity $\left\langle S^{H_{M}}\left(\mathbf{k}^{\prime}|\mathbf{p}| \mathbf{q}\right)\right\rangle$ of Eq. (39) simplifies to

$$
\begin{aligned}
\left\langle S^{H_{M}}\left(\mathbf{k}^{\prime}|\mathbf{p}| \mathbf{q}\right)\right\rangle= & \frac{1}{2} \Re\left[\epsilon_{i j m}\left\langle b_{i}\left(k^{\prime}\right) u_{j}(p) b_{m}(q)\right\rangle\right. \\
& -\epsilon_{j l m} \frac{k_{i} k_{l}}{k^{2}}\left\langle u_{i}(q) b_{m}\left(k^{\prime}\right) b_{j}(p)\right\rangle \\
& \left.+\epsilon_{j l m} \frac{k_{i} k_{l}}{k^{2}}\left\langle b_{i}(q) b_{m}\left(k^{\prime}\right) u_{j}(p)\right\rangle\right],
\end{aligned}
$$

which is computed perturbatively to the first order. The corresponding Feynman diagrams are

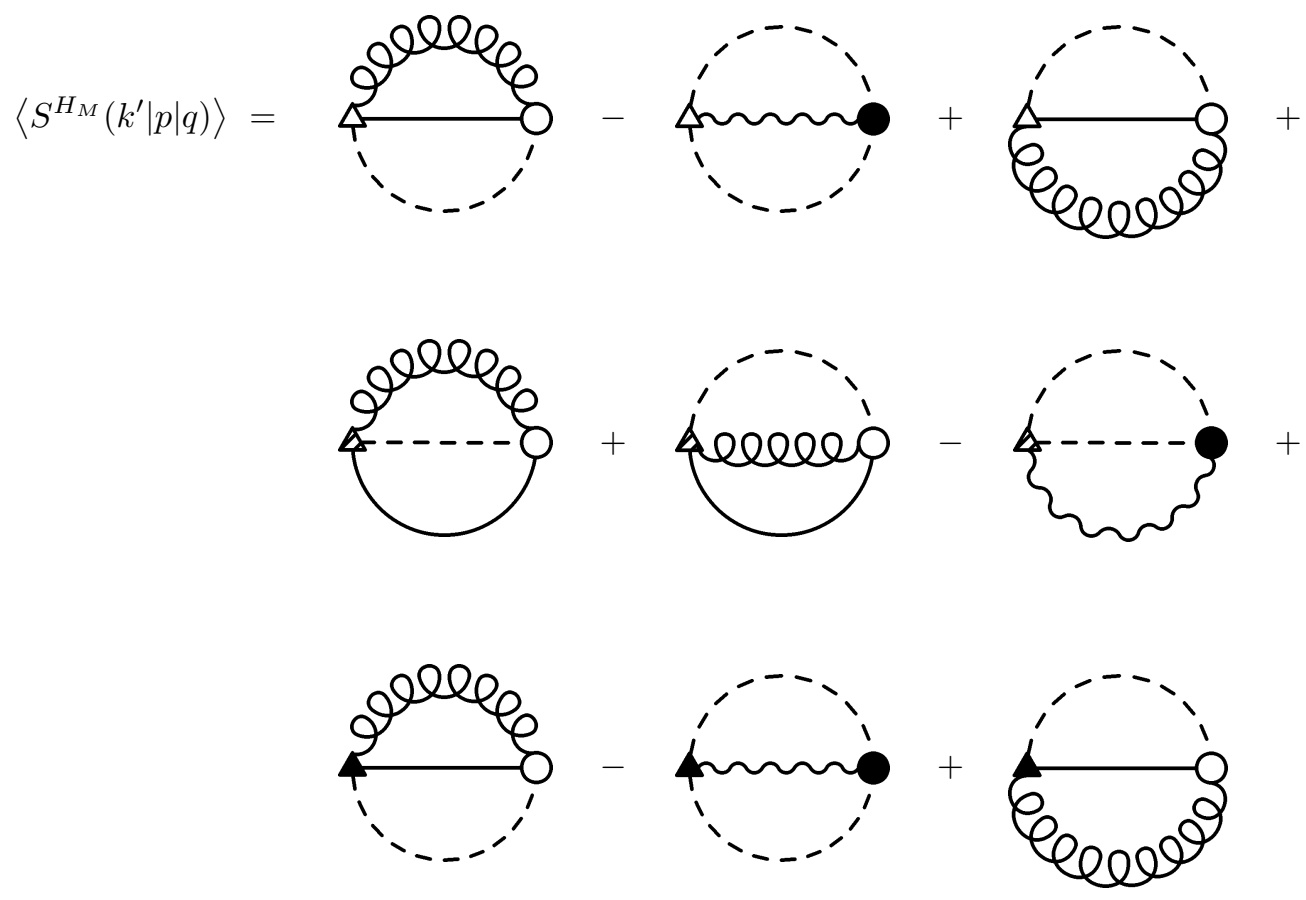

Here empty, shaded, and filled triangles (vertices) represent $\epsilon_{i j m},-\epsilon_{i j m} k_{i} k_{l} / k^{2}$ and $\epsilon_{i j m} k_{i} k_{l} / k^{2}$ respectively. The empty and filled circles (vertices) denote $(-i / 2) P_{i j m}^{-}$and $-i P_{i j m}^{+}$respectively. The solid, dashed, wiggly (photon), 
and curly (gluons) lines denote $\left\langle u_{i} u_{j}\right\rangle,\left\langle b_{i} b_{j}\right\rangle, G^{u u}$, and $G^{b b}$ respectively. When we substitute $\left\langle u_{i} u_{j}\right\rangle,\left\langle b_{i} b_{j}\right\rangle$ using Eqs. (1815), we obtain terms involving $C^{X}\left(p, t, t^{\prime}\right) H_{(M, K)}\left(q, t, t^{\prime}\right)$. The resulting expression for $\left\langle S^{H_{M}}\left(\mathbf{k}^{\prime}|\mathbf{p}| \mathbf{q}\right)\right\rangle$ is

$$
\begin{aligned}
& \left\langle S^{H_{M}}\left(\mathbf{k}^{\prime}|\mathbf{p}| \mathbf{q}\right)\right\rangle=\int_{-\infty}^{t} d t^{\prime}(2 \pi)^{3}\left[T_{31}(k, p, q) G^{b b}\left(k, t-t^{\prime}\right) \frac{H_{K}\left(p, t-t^{\prime}\right)}{p^{2}} C^{b}\left(q, t-t^{\prime}\right)\right. \\
& +T_{32}(k, p, q) G^{b b}\left(k, t-t^{\prime}\right) C^{u u}\left(p, t-t^{\prime}\right) H_{M}\left(q, t-t^{\prime}\right) \\
& +T_{33}(k, p, q) G^{u u}\left(p, t-t^{\prime}\right) H_{M}\left(k, t-t^{\prime}\right) C^{b b}\left(q, t-t^{\prime}\right) \\
& +T_{34}(k, p, q) G^{u u}\left(p, t-t^{\prime}\right) C^{b b}\left(k, t-t^{\prime}\right) H_{M}\left(q, t-t^{\prime}\right) \\
& +T_{35}(k, p, q) G^{b b}\left(q, t-t^{\prime}\right) H_{M}\left(k, t-t^{\prime}\right) C^{u u}\left(p, t-t^{\prime}\right) \\
& +T_{36}(k, p, q) G^{b b}\left(q, t-t^{\prime}\right) C^{b b}\left(k, t-t^{\prime}\right) \frac{H_{K}\left(p, t-t^{\prime}\right)}{p^{2}} \\
& +T_{37}(k, p, q) G^{b b}\left(k, t-t^{\prime}\right) H_{M}\left(p, t-t^{\prime}\right) C^{u u}\left(q, t-t^{\prime}\right) \\
& +T_{38}(k, p, q) G^{b b}\left(k, t-t^{\prime}\right) C^{b b}\left(p, t-t^{\prime}\right) \frac{H_{K}\left(q, t-t^{\prime}\right)}{q^{2}} \\
& +T_{39}(k, p, q) G^{b b}\left(p, t-t^{\prime}\right) H_{M}\left(k, t-t^{\prime}\right) C^{u u}\left(q, t-t^{\prime}\right) \\
& +T_{40}(k, p, q) G^{b b}\left(p, t-t^{\prime}\right) C^{b b}\left(k, t-t^{\prime}\right) \frac{H_{K}\left(q, t-t^{\prime}\right)}{q^{2}} \\
& +T_{41}(k, p, q) G^{u u}\left(q, t-t^{\prime}\right) H_{M}\left(k, t-t^{\prime}\right) C^{b b}\left(p, t-t^{\prime}\right) \\
& \left.+T_{42}(k, p, q) G^{u u}\left(q, t-t^{\prime}\right) C^{b b}\left(k, t-t^{\prime}\right) H_{M}\left(p, t-t^{\prime}\right)\right\} \\
& +T_{43}(k, p, q) G^{b b}\left(k, t-t^{\prime}\right) \frac{H_{K}\left(p, t-t^{\prime}\right)}{p^{2}} C^{b b}\left(q, t-t^{\prime}\right) \\
& \left.+T_{44}(k, p, q) G^{b b}\left(k, t-t^{\prime}\right) C^{u u}\left(p, t-t^{\prime}\right) H_{M}\left(q, t-t^{\prime}\right)\right\} \\
& +T_{45}(k, p, q) G^{u u}\left(p, t-t^{\prime}\right) H_{M}\left(k, t-t^{\prime}\right) C^{b b}\left(q, t-t^{\prime}\right) \\
& +T_{46}(k, p, q) G^{u u}\left(p, t-t^{\prime}\right) C^{b b}\left(k, t-t^{\prime}\right) H_{M}\left(q, t-t^{\prime}\right) \\
& +T_{47}(k, p, q) G^{b b}\left(q, t-t^{\prime}\right) H_{M}\left(k, t-t^{\prime}\right) C^{u u}\left(p, t-t^{\prime}\right) \\
& \left.+T_{48}(k, p, q) G^{b b}\left(q, t-t^{\prime}\right) C^{b b}\left(k, t-t^{\prime}\right) \frac{H_{K}\left(p, t-t^{\prime}\right)}{p^{2}}\right]
\end{aligned}
$$

The terms $T_{31 . .48}(k, p, q)$ can be obtained in terms of antisymmetric tensors $\epsilon_{j a l}, P_{i j m}^{ \pm}$etc. They have not been listed here due to lack of space.

[1] H. K. Moffatt, Magnetic Fields Generation in Electrically Conducting Fluids (Cambridge University Press, Cambridge, 1978).

[2] F. Krause and K. H. Rädler, Mean-Field Magnetohydrodynamics and Dynamo Theory (Pergamon Press, Oxford, 1980).

[3] M. K. Verma, Pramana (accepted for publication), nlin.CD/0103033 (2002).

[4] M. Steenbeck, F. Krause, and K.-H. Räadler, Z. Naturforsh 21a, 369 (1966).

[5] A. Pouquet, U. Frisch, and J. Léorat, J. Fluid Mech. 77, 321 (1976).

[6] A. V. Gruzinov and P. H. Diamond, Phys. Rev. Lett. 72, 1651 (1994).

[7] G. B. Field, E. G. Blackman, and H. Chou, Astrophys. J. 513, 638 (1999).

[8] H. Chou, Astrophys. J. 552, 803 (2000).

[9] A. Basu and J. K. Bhattacharjee, cond-mat/9812090 (1998).

[10] A. Basu, nlin.CD/0208010 (2002).

[11] S. Sridhar and P. Goldreich, Astrophys. J. 432, 612 (1994).

[12] P. Goldreich and S. Sridhar, Astrophys. J. 438, 763 (1995).

[13] M. K. Verma, Phys. Plasma 6, 1455 (1999).

[14] M. K. Verma, Phys. Rev. E 64, 26305 (2001).

[15] M. K. Verma, Phys. Plasma 8, 3945 (2001).

[16] M. K. Verma et al., J. Geophys. Res. 101, 21619 (1996).

[17] W. C. Müller and D. Biskamp, Phys. Rev. Lett. 84, 475 (2000).

[18] D. Biskamp and W. C. Müller, Phys. Plasma 7, 4889 (2000).

[19] A. Pouquet and G. S. Patterson, J. Fluid Mech. 85, 305 (1978).

[20] A. Brandenburg, Astrophys. J. 550, 824 (2001). 
[21] P. Frick and D. Sokoloff, Phys. Rev. E 57, 4155 (1998).

[22] G. Dar, M. K. Verma, and V. Eswaran, Physica D 157, 207 (2001).

[23] M. Meneguzzi, U. Frisch, and A. Pouquet, Phys. Rev. Lett. 47, 1060 (1981).

[24] R. M. Kulsrud and S. W. Anderson, Astrophys. J. 396, 606 (1992).

[25] U. Frisch, A. Pouquet, J. Léorat, and A. Mazure, J. Fluid Mech. 68, 769 (1975).

[26] W. D. McComb, The Physics of Fluid Turbulence (Claredon, Oxford University Press, 1990).

[27] Y. Zhou, Phys. Rev. A 41, 5683 (1990).

[28] V. Yakhot and S. A. Orszag, J. Sci. Comput. 1, 3 (1986).

[29] D. C. Leslie, Development in the Theory of Turbulence (Claredon, Oxford University Press, 1973).

[30] R. H. Kraichnan, J. Fluid Mech. 59, 745 (1973).

[31] A. A. Schekochihin, S. A. Boldyrev, and R. M. Kulsrud, astro-ph/0103333 (2001). 
TABLE I: The values of $I_{Y}^{X}=\left(\Pi_{Y}^{X} / \Pi\right) /\left(K^{u}\right)^{1.5}$ calculated using Eqs. 4748) for Alfvén ratios $r_{A}=1$ and $r_{A}=5000$.

\begin{tabular}{lcc}
\hline \hline & $r_{A}=1$ & $r_{A}=5000$ \\
\hline$I_{u>}^{u<}$ & $0.19-0.10 r_{K}^{2}$ & $0.53-0.28 r_{K}^{2}$ \\
$I_{b>}^{u<}$ & $0.62+0.3 r_{M}^{2}+0.095 r_{K} r_{M}$ & $1.9 \times 10^{-4}+1.4 \times 10^{-9} r_{M}^{2}+2.1 \times 10^{-5} r_{K} r_{M}$ \\
$I_{u>}^{b>}$ & $0.18-2.04 r_{M}^{2}+1.93 r_{K} r_{M}$ & $-5.6 \times 10^{-5}-1.1 \times 10^{-7} r_{M}^{2}+5.4 \times 10^{-4} r_{K} r_{M}$ \\
$I_{b>}^{b<}$ & $0.54-1.9 r_{M}^{2}+2.02 r_{K} r_{M}$ & $1.4 \times 10^{-4}-1.02 \times 10^{-7} r_{M}^{2}+5.4 \times 10^{-4} r_{K} r_{M}$ \\
$I_{b<}^{u<}$ & - & $-4.1 \times 10^{-3} r_{M}+8.1 \times 10^{-5} r_{K}$ \\
$I_{H_{M}}$ & $-25 r_{M}+0.35 r_{K}$ & -4 \\
\hline \hline
\end{tabular}

TABLE II: The values of energy flux ratios $\Pi_{Y}^{X} / \Pi$ for various values of $r_{A}, r_{K}$, and $r_{M}$ for $k^{-5 / 3}$ region. The first and second entries are nonhelical and helical contributions respectively.

\begin{tabular}{lcccc}
\hline \hline$\left(r_{A}, r_{K}, r_{M}\right)$ & $\Pi_{u>}^{u<} \Pi \Pi$ & $\Pi_{b>}^{u<} / \Pi$ & $\Pi_{u>}^{b<} / \Pi$ & $\Pi_{b>}^{b<} / \Pi$ \\
\hline$(5000,0.1,-0.1)$ & $(1.0,-0.0053)$ & $\left(3.2 \times 10^{-4}\right.$, & $\left(-9.7 \times 10^{-7}\right.$, & $\left(2.5 \times 10^{-4}\right.$, \\
& & $\left.-3.7 \times 10^{-7}\right)$ & $\left.-9.0 \times 10^{-6}\right)$ & $\left.-9.4 \times 10^{-6}\right)$ \\
$(5000,0.1,0.1)$ & $(1.0,-0.0053)$ & $\left(3.2 \times 10^{-4}\right.$, & $\left(-9.7 \times 10^{-5}\right.$, & $\left(2.5 \times 10^{-4}\right.$, \\
& & $\left.3.7 \times 10^{-7}\right)$ & $\left.9.0 \times 10^{-6}\right)$ & $\left.9.4 \times 10^{-6}\right)$ \\
$(1,0.1,-0.1)$ & $\left(0.13,-6.9 \times 10^{-4}\right)$ & $\left(0.43,-4.4 \times 10^{-4}\right)$ & $(0.13,-0.027)$ & $(0.37,-0.027)$ \\
$(1,0.1,0.1)$ & $\left(0.12,-6.5 \times 10^{-4}\right)$ & $\left(0.4,8.1 \times 10^{-4}\right)$ & $\left(0.12,-7.7 \times 10^{-4}\right.$ & $\left(0.35,8.3 \times 10^{-4}\right.$ \\
$(1,1,-1)$ & $(0.029,-0.015)$ & $\left(0.095,-9.9 \times 10^{-3}\right)$ & $(0.028,-0.61)$ & $(0.083,-0.60)$ \\
$(1,1,1)$ & $(0.12,-0.064)$ & $(0.39,0.079)$ & $(0.12,-0.075)$ & $(0.34,0.081)$ \\
$(1,0,1)$ & $(0.081,0)$ & $(0.26,0.013)$ & $(0.078,-0.86)$ & $(0.23,-0.8)$ \\
\hline \hline
\end{tabular}

